\title{
ADMINISTRATIVE BUCHHALTUNGSBERATUNG: EINE NEUE ART, EIN UNTERNEHMEN DURCH TECHNOLOGIE ZU FÜHREN
}

\section{ORIGINALER ARTIKEL}

SUMAR, Ramiro Rodrigues ${ }^{1}$

SUMAR, Ramiro Rodrigues. Administrative Buchhaltungsberatung: eine neue Art, ein Unternehmen durch Technologie zu führen. Revista Científica Multidisciplinar Núcleo do Conhecimento. Jahrgang 06, Ed. 07, Vol. 12, S. 91-104. Juli 2021. ISSN: 2448-0959, Zugangslink: https://www.nucleodoconhecimento.com.br/business-administration-de/technologiezu-fuehren, DOI: 10.32749/nucleodoconhecimento.com.br/business-administrationde/technologie-zu-fuehren

\section{ZUSAMMENFASSUNG}

Ziel: Beschreibung der Auswirkungen der Hindernisse und Potenziale, die die Technologie in die Buchhaltungsberatung gebracht hat. Frage: Wie kann die administrative Buchhaltungsberatung einem Unternehmen helfen, Technologien einzusetzen? Methoden: Die Studie ist eine bibliographische Übersicht. Eine Suche wurde mit den Deskriptoren durchgeführt: Technologie; Innovation; Buchhaltung; Abrechnung auf der Datenplattform von Spell (Scientific Periodicals Eletronic Library) im Juni 2021. Die Suche ergab 10 Artikel. Ergebnisse: die Ergebnisse als Hindernisse gebracht: Der zunehmende technologische Fortschritt, Anpassung an den Einsatz dieser neuen Technologien, Zentralität der Rolle des Buchhalters in traditionellen Modellen, Mangel an Softwarewissen,Mangel an spezialisierten Arbeitskräften, Fehlfunktion der Verbindung, Qualifizierte Arbeitskräfte. Und als Potentialitäten: Einsatz verschiedener digitaler Technologien, Agilität und Zeitgewinn,

\footnotetext{
${ }^{1}$ Master-Abschluss in Controllership, Spezialist für Technologiemanagement, Strategische Verwaltung Informationssicherheit und Abschluss in Rechnungswissenschaften.
}

RC: 93888

Disponível em: https://www.nucleodoconhecimento.com.br/business-administration- 
Bequemlichkeit, untrennbare Beziehung zwischen Technologie und beratender Buchhaltung Förderung der agilen und qualitativ hochwertigen Unterstützung für die verschiedenen Benutzer, Kundenzufriedenheit, Geschwindigkeit und Flexibilität und Kostensenkung. Schlussfolgerungen: Es versteht sich, dass Informationstechnologien die Ausübung der konsultativen Rechnungslegung positiv beeinflussen, obwohl sie zusätzlich zu den Vorteilen ihre Nachteile haben. Es ist erwähnenswert, dass die Technologien die Kontrolle von Buchhaltungsinformationen für den Ein- und Ausstieg von Materialien und Vorleistungen sowie deren Ursprung und Endziel ermöglichen und unnötige Verluste, einschließlich Abweichungen, Agilität, Flexibilität und Sicherheit, vermeiden.

Schlüsselwörter: Beratung, Innovation, Technologische Mittel, Informationssicherheit.

\section{EINLEITUNG}

Die Verbindung zwischen Buchhaltung und Technologien hat sich seit Jahrzehnten fortgesetzt, da die Buchhaltung nicht nur Wissenschaft ist, sondern auch eines der Informationssysteme, die in der älteren Unternehmensführung verwendet werden, was die Buchhaltung der Vergangenheit und die Buchhaltung der Gegenwart unterschiedlich macht, sind die Wege, in denen sie zur Unternehmensführung führen (FERREIRA et al., 2017).

Die Technologie hat sich im Laufe der Jahre weiterentwickelt und innovationiert, so dass das Bedürfnis nach dem Menschen erfüllt wird. Da es sich um ein unbegrenztes Werkzeug handelt, bringt die Entwicklung von Technologien in Kombination mit der Buchhaltung relevante Beiträge zum Wachstum von Organisationen (SILVA et al., 2020).

Die kontinuierliche Nutzung von Computernetzwerken und die Kombination verschiedener Rechnungslegungsinstrumente begünstigen die Speicherung, Registrierung, Verarbeitung und erleichtern die Realisierung der Kreuzung von

RC: 93888

Disponível em: https://www.nucleodoconhecimento.com.br/business-administration- 
Informationen immer schneller, was eine sicherere Entscheidungsfindung innerhalb der Organisation ermöglicht (MARION; RIBEIRO, 2017; DE ANDRADE SIMÕES et al., 2021).

Derzeit war die Suche nach Informationen nützlich und gleichzeitig relevant für das Wachstum der Organisation. Der Transfer von Informationen in Wissen, sowie die rechtzeitige Anwendung von Wissen in der Praxis und die Verbreitung dieser Handlungen für die Entwicklung eines Unternehmens, sind Potenziale der Technologie zugunsten der beratenden Rechnungslegung für den Unternehmer.

Viele Buchhalter verwenden dieses Tool jedoch nicht, um Raum und Zeit zu gewinnen und ihre Kunden zu binden, was deutlich macht, dass in einigen Situationen die Technologien für die Zähler nicht verwendet werden. In diesem Praktikum, das die Problematisierung der fraglichen Studie identifiziert, gibt es die Frage, die das Thema dieser Studie leitete: Wie kann die administrative Buchhaltungsberatung einem Unternehmen helfen, Das Technologien einsetzt?

Der aktuelle Arbeitsmarkt motiviert die Menschen oft, sich in ihrer beruflichen Tätigkeit neu zu formulieren, da das universitäre Grundbildungssystem und das neue Wissen angesichts der Praxis, die der Student nach dem Abschluss absolvieren wird, immer noch gering werden.

Laut Villa et al (2012, S.107) "haben traditionelle Buchhalter die Ansicht, dass Unternehmer nicht das Wissen suchen, das für die Verwaltung ihres Unternehmens erforderlich ist, dh sie nehmen keine Verbesserungskurse oder Schulungen in der Verwaltung". Um dieses Zitat des Autors zu paraphrasieren, weist darauf hin, dass der Unternehmer nur das Interesse hat, die Anforderungen der Steuern zu erfüllen, wodurch der Wettbewerbsmarkt vergessen wird, was dazu führt, dass das Unternehmen Platz verliert und sich nicht in seiner Qualität entwickelt.

RC: 93888

Disponível em: https://www.nucleodoconhecimento.com.br/business-administration- 
In dieser Platzierung wird die Bedeutung des Beratungszählers beobachtet. Diese Arbeit wird zur Begründung des Forschers hinzugefügt, zu verstehen, warum viele Buchhalter keine beratende Buchhaltung verwenden, um mit ihren Kunden zu arbeiten, da dieses Hilfsinstrument insbesondere in den Pandemiezeiten des neuen Coronavirus Einfluss ausübt, so dass das Unternehmen nicht Gefahr läuft, sich auf dem Wettbewerbsmarkt für sein Einkommen zu engagieren.

Daher wird das Thema relevant, denn mit dem Aufkommen von Technologien hat die Buchhaltung von Unternehmen eine andere Richtung einzuschlagen als die Buchhaltung vor Jahrzehnten und damit hat der Buchhalter jetzt die Verantwortung und Verpflichtung, seinen Beruf von der Gesellschaft und den Unternehmern als einen Beruf zu identifizieren, der sich an technologische Mittel anpasst, um mehr Sicherheit und Zuverlässigkeit für Organisationen zu gewährleisten.

Daher hat diese Arbeit als allgemeines Ziel: Die Auswirkungen der Hindernisse und Potenziale zu beschreiben, die die Technologie in die Buchhaltungsberatung gebracht hat.

\section{METHODISCHER PROZESS}

Diese Studie ist eine systematische bibliographische Übersicht qualitativer Natur. Laut Sampaio und Mancini (2007) wird diese Art von Studie durch die Suche nach Artikeln durchgeführt, die für das untersuchte Thema relevant sind, basierend auf der Synthese relevanter Themen in Kombination mit systematisierten Untersuchungsmethoden, kritischer Analyse, Zusammenfassung der ausgewählten Informationen und wichtigen Problemen, die weitere Studien erfordern.

Zur Durchführung der Forschung wurden die folgenden von Sampaio und Mancini (2007) empfohlenen Schritte befolgt: Formulierung der Leitfrage der Studie; Studienort; Kritische Auswertung von Studien; Datenerhebung; Datenanalyse; Ethische Aspekte.

RC: 93888

Disponível em: https://www.nucleodoconhecimento.com.br/business-administration- 
Aus der Konstruktion einer Leitfrage ergeben sich einige Annahmen, die diese Studie leiten und / oder abgrenzen, so dass die Anwendbarkeit von Technologien im Universum der Abfrage der Zahlen und Werte, in die der Buchhalter eingefügt wird, hervorgehoben werden kann. Die Frage, die das Thema leitete, war: Wie kann die administrative Buchhaltungsberatung einem Unternehmen helfen, Technologien einzusetzen?

Das Material wurde in den Datenbanken Spell (Scientific Periodicals Eletronic Library) ausgewählt. Die Studienstichprobe bestand aus Artikeln (Originalforschung; Übersichtsartikel; Monographien, Abschlussarbeiten und Dissertationen). Als Einschlusskriterien wurden von 2016 bis 2020 Artikel in portugiesischer und englischer Sprache heran herangeordnet, die vollständig und kostenlos zur Verfügung stehen. Alle Artikel, die in diese Kriterien eingerahmt wurden, die in ihrem Wissensgebiet das Rechnungswesen abdeckten und als Titel oder Abstract Themen hatten, die für den Einsatz von Technologien relevant waren, wurden für die Forschung gerahmt. Andere Artikel, die nicht enthalten oder dupliziert wurden, wurden ausgeschlossen. Die Schlüsselwörter, die für die Suche nach diesen Artikeln in Spell verwendet wurden, waren: Technologie; Innovation; Buchhaltung; beratende Buchhaltung.

Die kritische Bewertung der Produktionen, die ausgewählt und in die Studie aufgenommen wurden, führte zu Interpretationen und Synthesen der Ergebnisse, wobei die in jeder Studie vorhandenen wissenschaftlichen Informationen und die relevanten Faktoren für die betreffende Studie identifiziert wurden, die Richtlinien für die zukünftige Forschung nahelegen. Um die Studie zu validieren, macht der Forscher deutlich, welche Lücken in der Studie gefunden wurden und welche zukünftigen Wege andere Forscher in ihrer wissenschaftlichen Forschung einschlagen können. Die Hauptinformationen wurden in Tabellen angeordnet, um die für die Ergebnisse der Artikel relevanten Informationen deutlich zu machen und die Themen mit der für das Thema relevanten Literatur zu verknüpfen.

RC: 93888

Disponível em: https://www.nucleodoconhecimento.com.br/business-administration- 
Die Daten wurden im Juni 2021 erhoben. Die Sammlung erfolgte unter Einbeziehung der Ein- und Ausschlusskriterien der Artikel. Betonen Sie auch, dass die Forschung auf der Grundlage der durchgeführten Analyse auch versucht, über die beratende Rechnungslegung und den Einsatz von Technologien zu berichten. Es wurden zwei Suchen durchgeführt, die die Schlüsselwörter einstreuten.

Die in der veröffentlichten Literatur gefundenen Daten erfolgten nach Ercoles Vorschlag; Melo und Alcoforado (2014), in denen der Gutachter versuchen sollte, die Ergebnisse unparteiisch zu bewerten und Erklärungen für die unterschiedlichen oder widersprüchlichen Ergebnisse in den verschiedenen Studien zu suchen.

\section{ANALYSE DER ERGEBNISSE UND DISKUSSIONEN}

Die Suchanfragen ergaben insgesamt 343 Ergebnisse mit den Keywords. Alle Titel und Abstracts wurden gelesen und Artikel, die den Zielen der betreffenden Studie entsprechen konnten, wurden entfernt, was zu 10 Studien führte.

So präsentieren wir ein Bild mit dem Titel des Artikels, Autoren, Erscheinungsjahr (Referenz), Ziel der Studie und gefundenen Ergebnissen.

Tabelle 1 - Ausgewählte Artikel

\begin{tabular}{|c|c|c|}
\hline $\begin{array}{l}\text { Titel/Referenz } \\
\text { des Artikels }\end{array}$ & Ziele & Befund \\
\hline $\begin{array}{l}\text { No caminho da } \\
\text { inovação: } \\
\text { análise das } \\
\text { capacidades de } \\
\text { inovação de } \\
\text { empresas } \\
\text { contábeis }\end{array}$ & $\begin{array}{l}\text { Untersuchen Sie die } \\
\text { Innovationsfähigkeit von } \\
\text { Buchhaltungsbüros auf dem } \\
\text { brasilianischen Markt bei der } \\
\text { Verwendung digitaler } \\
\text { Technologien, basierend auf den } \\
\text { Fähigkeiten }\end{array}$ & $\begin{array}{l}\text { Die Buchhaltung beginnt auf } \\
\text { dem traditionellen Weg der } \\
\text { digitalen Innovation und zeigt } \\
\text { die Qualität und den Wert, } \\
\text { den technologische } \\
\text { Lösungen generieren } \\
\text { können, wenn sie in der }\end{array}$ \\
\hline
\end{tabular}

RC: 93888

Disponível em: https://www.nucleodoconhecimento.com.br/business-administration- 


\begin{tabular}{|c|c|c|}
\hline $\begin{array}{l}\text { diante das } \\
\text { tecnologias } \\
\text { digitais }\end{array}$ & $\begin{array}{l}\text { Technologieentwicklung, des } \\
\text { Betriebs, des Managements und } \\
\text { der Transaktion. }\end{array}$ & $\begin{array}{l}\text { Wirtschaft und insbesondere } \\
\text { in prozessen genutzt } \\
\text { werden.Diese Zunahme der } \\
\text { Technologie führt zu } \\
\text { Veränderungen in den } \\
\text { Geschäftsmodellen } \\
\text { Buchhaltung. }\end{array}$ \\
\hline $\begin{array}{l}\text { Indústria } 4.0 \text { e } \\
\text { avanços } \\
\text { tecnológicos da } \\
\text { área contábil: } \\
\text { perfil, } \\
\text { percepções e } \\
\text { expectativas } \\
\text { dos } \\
\text { profissionais } \\
\text { Xavier; Carraro } \\
\text { und Rodrigues } \\
\text { (2020). }\end{array}$ & $\begin{array}{l}\text { Analysieren Sie das Profil des } \\
\text { Buchhaltungsprofis angesichts } \\
\text { des technologischen Fortschritts } \\
\text { und untersuchen Sie, wie er sich } \\
\text { in Bezug auf neue Trends verhält. }\end{array}$ & $\begin{array}{l}\text { Die meisten Fachleute sind } \\
\text { daran interessiert, sich an } \\
\text { neue Technologien } \\
\text { anzupassen, um die } \\
\text { Anforderungen der Funktion } \\
\text { zu erfüllen. Allerdings gab es } \\
\text { einige Widerstände gegen die } \\
\text { Veränderungen, vor allem bei } \\
\text { Fachkräften unter } 35 \text { Jahren } \\
\text { und } \\
\text { Unternehmensbereich. }\end{array}$ \\
\hline $\begin{array}{l}\text { Características } \\
\text { dos Diferentes } \\
\text { Modelos de } \\
\text { Negócios } \\
\text { Contábeis em } \\
\text { Relação às } \\
\text { Áreas da } \\
\text { Contabilidade }\end{array}$ & $\begin{array}{l}\text { Identifizieren Sie den Wert, der } \\
\text { dem kunden von } \\
\text { Buchhaltungsdienstleistungen } \\
\text { durch die strukturelle } \\
\text { Organisation und die Merkmale } \\
\text { traditioneller Geschäftsmodelle in } \\
\text { Bezug auf ihre jeweiligen } \\
\text { Tätigkeitsbereiche in der } \\
\text { Buchhaltung geliefert wird. }\end{array}$ & $\begin{array}{l}\text { Der Buchhaltungsbereich Mit } \\
\text { dem Schwerpunkt } \\
\text { Informationssysteme basiert } \\
\text { auf der Beratung in den } \\
\text { Bereichen } \\
\text { Datenverarbeitung, } \\
\text { Informationstechnologie und } \\
\text { Betriebssysteme. }\end{array}$ \\
\hline
\end{tabular}

RC: 93888

Disponível em: https://www.nucleodoconhecimento.com.br/business-administration- 
Behr (2020).

As

Contribuições

da

Contabilidade

Consultiva: Um

Estudo de

Caso/The

Contributions

of Advisory

Accounting: A

Case Study

Angelim und

Barreto (2020).

Überprüfen Sie die Beiträge, die durch den Fortschritt der die Technologie in der Buchhaltung sowie in der beratenden Buchhaltung gefördert werden.

die Bedeutung der beratenden

Rechnungslegung im aktuellen Szenario sowie die wesentliche Rolle der Technologie in der Buchhaltung wahrnehmen. $\mathrm{Zu}$ den Herausforderungen, mit denen sich die Befragten befassen, gehören die Anpassung an den Einsatz neuer technologischer Werkzeuge, die Notwendigkeit, Strategien zu skizzieren, um dem Kunden besser zu dienen, und die Notwendigkeit, das Management und das Geschäft jedes Kunden zu kennen.

\begin{tabular}{|l|l|l|}
\hline Impact & Bestimmen Sie die Auswirkungen & Die Forschungsergebnisse \\
information & der Informationstechnologie auf & zeigten, dass die \\
technology on & die Buchhaltungslinie im globalen & Informationstechnologie \\
$\begin{array}{l}\text { accounting line } \\
\text { of works }\end{array}$ & System. & einen wesentlichen Einfluss \\
& & auf die Buchhaltung hat.
\end{tabular}

RC: 93888

Disponível em: https://www.nucleodoconhecimento.com.br/business-administrationde/technologie-zu-fuehren 


\section{Asuquo; Dan und Effiong (2020).}

The impact of Untersuchen Sie die Vorteile von information and IT-Anwendungen in der communication Buchhaltung. technology (ICT) in the accounting system: advantages, applications, and challenges

Al-Shafeay;

Al_Dujaili und

Al-Wattar

(2020).
Die Buchhaltung, wie die Sprache des Geschäfts und des Informationssystems, muss sich an neue Technologien anpassen, um den Nutzern von Finanzinformationen zu dienen. Im Informationszeitalter verändern sich die Marktanforderungen an Absolventen der Buchhaltung zunehmend.

The Impact of Identifizieren Sie, ob sich die Untersuchungen haben Information Technologie (positiv oder negativ) gezeigt, dass Technology on auf Buchhaltungsanwendungen The Quality of ausgewirkt hat und ob die Geschwindigkeit und Accounting Informationstechnologie in der technologischsten Information Buchhaltung einen Einfluss auf Auswirkungen auf (SFAC NO 8, die Qualität der Finanzberichte in Buchhaltungsanwendungen 2010) Saudi-Arabien hat. mit verbesserten und zuverlässigen Eigenschaften

Al Natour (2020). haben.
A Consultoria
Durchführung
einer Die

RC: 93888

Disponível em: https://www.nucleodoconhecimento.com.br/business-administration- 


\begin{tabular}{l|lr|l|} 
Contábil como & dokumentarischen & und & Wirtschaftsprüfungsberatung \\
Oportunidade & bibliometrischen Studie & zur & stell einen qualitativen \\
de Negócios & Rechnungslegungsberatung & im & finanziellen Renditefaktor für \\
Profissionais - & Zeitraum von 2006 bis 2016. & & Unternehmen dar und erhöht \\
Um Estudo & & & damit den Wettbewerbsvorteil \\
Documental & & & für sie.
\end{tabular}

Almeida et al (2018).

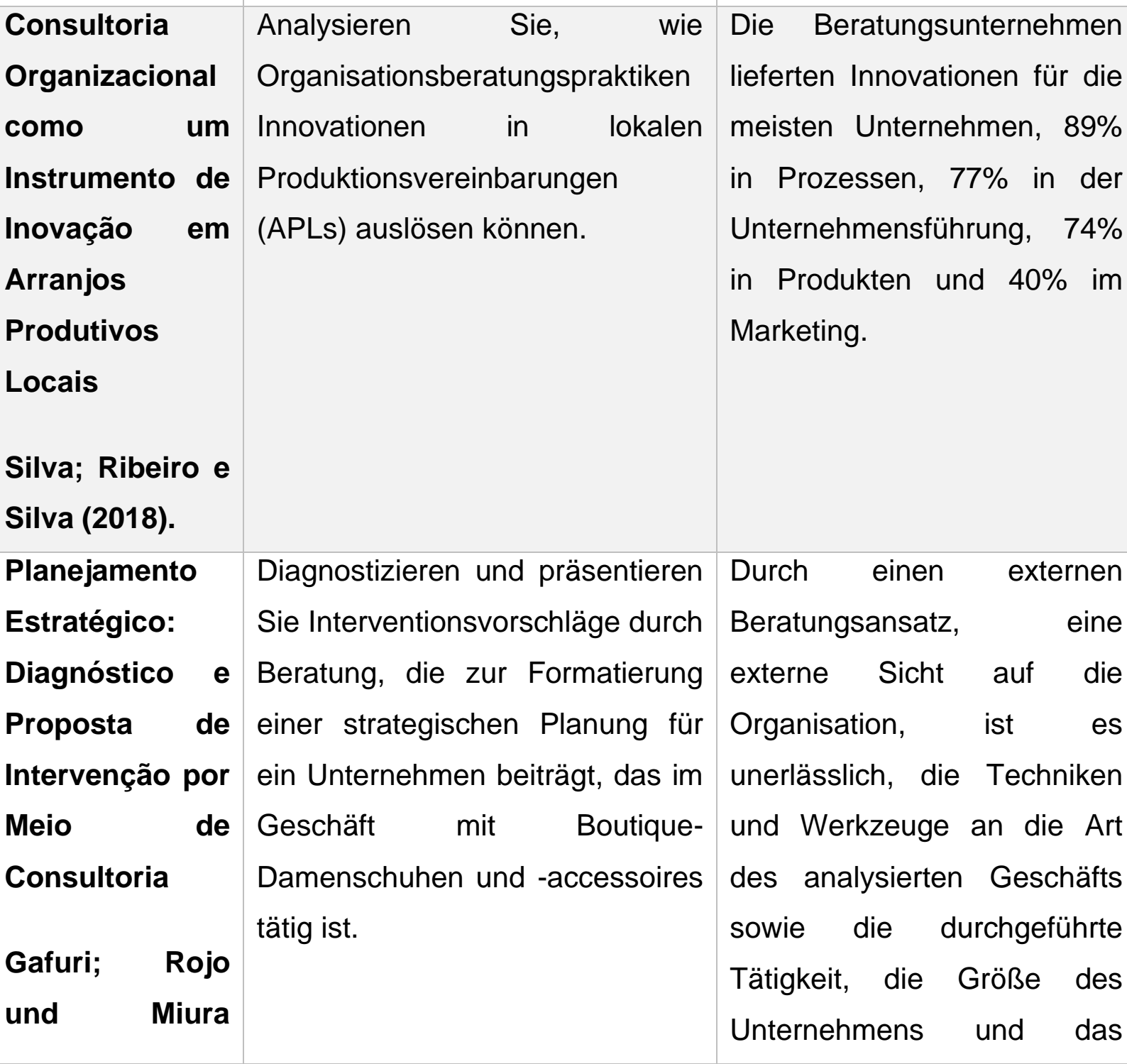

RC: 93888

Disponível em: https://www.nucleodoconhecimento.com.br/business-administration- 
(2017).

Verhandlungsvolumen

anzupassen.

Quelle: Eigene Autorenschaft

Die ausgewählten Artikel identifizierten Hindernisse für den Einsatz von Technologien durch den Zähler. Tabelle 2 enthält eine Synthese dieser Schwierigkeiten aus der Referenz des ausgewählten Artikels.

Tabelle 2 - Aufgetretene Hindernisse

\begin{tabular}{|c|c|}
\hline Artikeltitel, Referenz & Hindernisse \\
\hline $\begin{array}{l}\text { No caminho da inovação: análise } \\
\text { das capacidades de inovação de } \\
\text { empresas contábeis diante das } \\
\text { tecnologias digitais }\end{array}$ & Der wachsende technologische Fortschritt \\
\hline 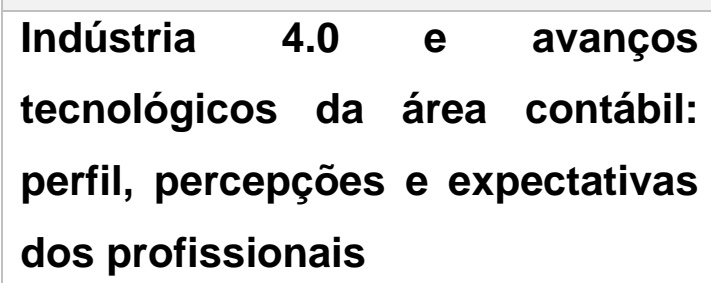 & $\begin{array}{l}\text { Anpassung an den Einsatz dieser neuen } \\
\text { Technologien }\end{array}$ \\
\hline $\begin{array}{l}\text { Características dos Diferentes } \\
\text { Modelos de Negócios Contábeis } \\
\text { em Relação às Áreas da } \\
\text { Contabilidade }\end{array}$ & $\begin{array}{l}\text { Die zentrale Rolle des Buchhalters in } \\
\text { traditionellen Geschäftsmodellen der } \\
\text { Buchhaltung und die technologischen } \\
\text { Ressourcen werden in diesem Szenario } \\
\text { immer noch unterstützt. }\end{array}$ \\
\hline $\begin{array}{llr}\text { As } & \text { Contribuições } & \text { da } \\
& & \end{array}$ & Anpassung an den Einsatz neuer \\
\hline 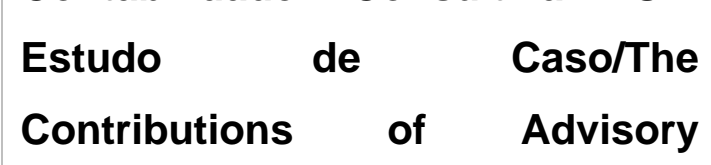 & \\
\hline Accounting: A Case Study & \\
\hline
\end{tabular}

RC: 93888

Disponível em: https://www.nucleodoconhecimento.com.br/business-administration- 
Impact of information technology Behalten Sie den erkennbaren Geschmack on accounting line of works Ihrer Vergangenheit, der in Charakter und Integrität verwurzelt ist

The impact of information and Unerfahrene Projektmanager; Mangel an communication technology (ICT) Softwarekenntnissen; Unvollständige in the accounting system: Verträge; Mangel an spezialisierten advantages, applications, and Arbeitskräften.

challenges

The Impact of Information Mittel zur Bewältigung von elektrischen Technology on The Quality of Problemen, Fehlfunktionen der Accounting Information (SFAC Internetverbindung und Computerviren. NO 8, 2010)

A Consultoria Contábil como Längere Laufzeit und Zuweisung größerer Oportunidade de Negócios Ressourcen und Ausführungskosten.

Profissionais - Um Estudo

Documental

Consultoria Organizacional como Facharbeiter.

um Instrumento de Inovação em

Arranjos Produtivos Locais

Planejamento Estratégico: Mangel an Softwarekenntnissen.

Diagnóstico e Proposta de

Intervenção por Meio de

Consultoria

Quelle: Eigene Autorenschaft

Es wird beobachtet, dass es mehrere Hindernisse gibt, die in den Artikeln nachgewiesen werden, wenn es um den Einsatz von Technologien für die Buchhaltungsberatung geht. Diese Hindernisse ergeben sich hauptsächlich aus den Schwierigkeiten vieler Fachleute, sich nicht an den Einsatz von Technologien in ihrer

$\mathrm{RC}: 93888$

Disponível em: https://www.nucleodoconhecimento.com.br/business-administration- 
Routine anzupassen, oder weil der Fachmann nicht richtig qualifiziert oder qualifiziert ist, oder aus Angst vor technologischen Veränderungen.

Schiavi et al (2020) weist darauf hin, dass Technologien als Unterstützung gesehen werden sollten, um insbesondere in Beratungsunternehmen gute Ergebnisse anzustreben, jedoch sollte sich der Buchhalter der technologischen Fortschritte bewusst sein, die nicht langsam sind, was es dem Fachmann ermöglicht, Angst zu haben, Technologie als gezielte Unterstützung für die Beratung zu nutzen.

Xavier; Carraro und Rodrigues (2020) erwähnen, dass die beratende Buchhaltung aus technologischen Mitteln gesteuert werden muss, da sie mehr Sicherheit, Präzision und Zuverlässigkeit in den gefundenen Daten begünstigt, aber damit dies geschieht, liegt es am Buchhalter, sich der Veränderungen des technologischen Fortschritts bewusst zu sein und sich an das gesamte System anzupassen, um dem Kunden fortschrittliche und erstklassige Technologien zu bringen.

Schiavi und Behr (2020) und Angelim und Barreto (2020) berichten, dass ein Teil der Buchhaltungsfachleute immer noch ihre Domäne in der Tradition der Rechnungslegungswissenschaft hat und die vollständige Präsenz von Technologien für die beste Qualität in der Buchhaltungsberatung nicht zulässt, was eine erhebliche Verzögerung bei der Entdeckung der Entscheidungsfindung widerspiegelt.

Es macht es notwendig, dass der Fachmann sich der neuen technologischen Trends zur Verbesserung des Unternehmens bewusst ist, ein Buchhalter, der in der Verwendung von Technologien geschult ist, neigt dazu, die Zeit zu verkürzen, um das Gleichgewicht des Unternehmens zu erreichen, sowie die Zuverlässigkeit in den gesammelten Daten zu übergeben.

Nach Asuquo; Dan und Effiong (2020) consultative Accounting bringt die Möglichkeit, in allen Bereichen der Buchhaltung zu expandieren, ob auf managementbezogene oder finanzielle Weise, was die Arbeit der Beratung bestimmt, ist der Grund, warum

RC: 93888

Disponível em: https://www.nucleodoconhecimento.com.br/business-administration- 
Beratung gearbeitet wird, aber die Verwurzelung der traditionellen Buchhaltung, schafft ein großes Hindernis zwischen dem Buchhalter und den Technologien, wo sich dieser Fachmann in Anwesenheit des Kunden in seinem Büro noch sicherer fühlt.

Das mangelnde Wissen über die Technologien und ihre Vorteile durchdringt immer noch das Leben vieler Fachleute. Mangelnde Berufsausbildung, mangelndes Wissen über Programme, qualifizierte Arbeitskräfte und andere Hindernisse ermöglichen es Technologien, nicht in die Buchhaltungsberatung einzudringen und so schnell, sicher und effektiv datenanalyse und folglich Entscheidungsfindung zu begünstigen ( $A L$ SHAFEAY; AL_DUJAILI; AL-WATTAR, 2020; ALMEIDA et al., 2018; SILVA; RIBEIRO; SILVA, 2018; GAFURI, ROJO; MIURA, 2017).

Die Hindernisse beziehen sich jedoch nicht nur auf die Schwierigkeiten der Buchhalter bei der Verwendung von Technologien, die Fehlfunktion von Programmen, die Langsamkeit des Internets, Viren im Computer können die Nichteignung des Fachmanns für das Medium der Technologien zur Unterstützung der beratenden Buchhaltung beeinflussen (AL NATOUR, 2020).

Die Verwendung von Softwares und Hardwares erfordert ein intensives Engagement des Fachmanns, um zu wissen, wie man die Tools und mit diesen gegenwärtigen Methoden verwendet, die dem Unternehmen niedrigere Kosten und größere Vorteile bringen können. Unter Berücksichtigung dieser Hindernisse in ihrer Komplexität des technologischen Umfelds werden die Potenziale identifiziert, die Technologien in die Buchhaltungsberatung einbringen können.

Tabelle 3 - Potenzial für Die Buchhaltungsberatung und den Einsatz von Technologien

Artikeltitel, Referenz

No caminho da inovação: Einsatz verschiedener digitaler Technologien;

RC: 93888

Disponível em: https://www.nucleodoconhecimento.com.br/business-administration- 
análise das capacidades de vollständig online handeln, um mehr inovação de empresas Zugänglichkeit und Agilität bei der Bereitstellung contábeis diante das von Buchhaltungsdienstleistungen zu erreichen tecnologias digitais

Indústria $\quad 4.0$ e avanços Agilität und Zeitgewinn. Annehmlichkeit. tecnológicos da área contábil: perfil, percepções e expectativas dos profissionais

Características dos Diferentes Die Verwendung der Verwendung der Modelos de Negócios technologischen Mittel des Benutzers wie EContábeis em Relação às Mails, Telefon, unter anderem zur Durchführung Áreas da Contabilidade von Buchhaltungsberatung.

As Contribuições da Untrennbare Beziehung zwischen Technologie Contabilidade Consultiva: Um und beratender Buchhaltung, die eine agile und Estudo de Caso/The qualitativ hochwertige Unterstützung für die Contributions of Advisory verschiedenen Benutzer fördert.

Accounting: A Case Study

Impact of information Befriedigen Sie Ihre internen und externen technology on accounting line Zielgruppen, indem Sie innen kontinuierlich und of works konsistent Qualitäts- und Leistungsdienstleistungen anbieten.

The impact of information and Kundenzufriedenheit; Agilität; Zuverlässigkeit. communication technology (ICT) in the accounting system: advantages, applications, and challenges

The Impact of Information Schnelligkeit und Flexibilität Technology on The Quality of Accounting Information (SFAC

RC: 93888

Disponível em: https://www.nucleodoconhecimento.com.br/business-administration- 
NO 8, 2010)

A Consultoria Contábil como Risikoanfällig für die erwarteten Ergebnisse der Oportunidade de Negócios durchzuführenden Arbeiten

Profissionais - Um Estudo

Documental

Consultoria Organizacional Neue Methoden der Arbeitsorganisation; Neue como um Instrumento de Techniken für das Wissensmanagement; Inovação em Arranjos Signifikante Veränderungen in den Beziehungen

Produtivos Locais zu anderen Unternehmen

Planejamento Estratégico: Kostensenkung, Managementkontrolle mit Diagnóstico e Proposta de Entfaltung für Einkaufspläne. Intervenção por Meio de Consultoria

Quelle: Eigene Autorenschaft

Es ist offensichtlich, dass die Notwendigkeit, in neue Konzepte und Kenntnisse einzutauchen, für Buchhaltungsfachleute von größter Bedeutung ist, da hierfür das Potenzial von Technologien in der Buchhaltungsberatung beobachtet wird. Die Buchhaltung wird als grundlegendes Instrument zur Unterstützung des Wachstums des Unternehmens angesehen, jedoch macht der Mangel an Kenntnissen der Technologien durch den Buchhalter dieses erworbene Wissen in der Traditionalität der Rollen identifiziert.

Schiavi et al. (2020) erwähnen mehrere Möglichkeiten, dass Technologien die Buchhaltungsberatung begünstigen können, das Zitat lautet: Einsatz verschiedener digitaler Technologien; vollständig online handeln, um mehr Zugänglichkeit und Agiliät bei der Bereitstellung von Buchhaltungsdienstleistungen zu erreichen. Diese Positionen der Autoren identifizieren die Bedeutung der Buchhaltung und der

RC: 93888

Disponível em: https://www.nucleodoconhecimento.com.br/business-administration- 
Technologien, die sich immer nebeneinander bewegen, was die Loyalität des Buchhalters gegenüber seinen Kunden begünstigt.

Xavier; Carraro und Rodrigues (2020) bestätigen mit den oben genannten Autoren die Potenziale von Technologien für die Buchhaltungsberatung und bekräftigen, dass neben diesen Platzierungen auch Agiliät, Zeitgewinn und Komfort sowohl des Unternehmens als auch des Buchhalters erwähnt werden können.

Für Schiavi und Behr (2020) bezieht sich der Einsatz von Technologien zur Durchführung von Buchhaltungsberatung nicht nur auf Fragen von Computerprogrammen, Telefon, sozialen Netzwerken, E-Mails und anderen Technologien, die dem traditionellen Buchhalter helfen können, seine Beratung durchzuführen, ohne unbedingt mit Programmen arbeiten zu müssen.

Laut Angelim und Barreto (2020) haben Technologien bereits ihre Beteiligung an der beratenden Buchhaltung, aber das Vorhandensein von Erleichternden Programmen für die Realisierung von Buchhaltungsberatung hat zu einer sogenannten Innovationstechnologie geführt, die Agiliät und mehr Zuverlässigkeit in die präsentierten Daten bringt.

Nach Asuquo; Dan und Effiong (2020) und Al-Shafeay; Al Dujaili und Al-Wattar (2020), beratende Buchhaltung zielt darauf ab, die zuverlässigsten Informationen über das Unternehmen und die erzielten Ergebnisse zu liefern, Kundenzufriedenheit durch Qualitäts- und Leistungsdienstleistungen zeigt den Unterschied der Anwesenheit der fortschrittlichsten Technologie für den Buchhalter.

Al Natour (2020), dass Technologien schnell und flexibel sind, zwei wesentliche Voraussetzungen für die Arbeit des Buchhalters, der in seiner Beraterzeit zum Feind für die Entscheidungsfindung wird, da der wettbewerbsorientierte Markt in Bezug auf Unternehmen immer anspruchsvoller wird.

RC: 93888

Disponível em: https://www.nucleodoconhecimento.com.br/business-administration- 
Nach Almeida et al. (2018) Trends zur Steigerung des Gewinns eines Unternehmens wirken sich direkt auf den Buchhalter aus, was das Wissen der Rechnungslegung über die Risikoübernahme vertieft. Die Technologien wirken, um weniger Risiko für den Buchhalter und eine bessere Rentabilität für das Unternehmen zu gewährleisten, was dem Kunden und dem Firmeninhaber einen qualitativ hochwertigen Service und in einer agilen Zeit ermöglicht.

Silva; Ribeiro e Silva (2018) e Gafuri; Rojo und Miura (2017) betonen, dass Technologien neben der Bereitstellung einer genauen und sicheren Analyse auch Methoden der Arbeitsorganisation, des Wissensmanagements, der besseren Beziehung zu anderen Unternehmen, der Kostenkontrolle, der Verbesserung der Entscheidungsfindung und anderer Möglichkeiten bieten, die auf eine höhere Produktivität mit niedrigeren Kosten im Vergleich zu traditionellen und manuellen Techniken abzielen.

Es wird angenommen, dass Technologien in Kombination mit der beratenden Buchhaltung mehrere Möglichkeiten haben, die es der Buchhaltung ermöglichen, ihre Entscheidungsfindung $\mathrm{zu}$ treffen, so dass das Unternehmen keine finanziellen Probleme hat oder den Wettbewerbsmarkt verlässt und auf dem nachgewiesenen Problem handelt.

\section{ENDGÜLTIGE ÜBERLEGUNGEN}

Die Hindernisse für den Einsatz von Technologien in der konsultativen Buchführung sind nach wie vor besorgniserregend, wenn man bedenkt, dass der Buchhalter als Hilfe für die Technologie zur Verbesserung seiner Dienstleistung dienen sollte und dass die Nichtakzeptanz des Einsatzes von Technologien dazu führen kann, dass dieser Fachmann von seinen Aufgaben disqualifiziert und diskreditiert wird.

Der Buchhaltungsexperte muss nachhaltige Praktiken im Auge behalten, um mehr finanzielle und betriebliche Vorteile für Unternehmen erzielen zu können, da neben

$\mathrm{RC}: 93888$

Disponível em: https://www.nucleodoconhecimento.com.br/business-administration- 
den hohen Kosten für den Erwerb und die Wartung des gesamten technologischen Installationsprozesses auch Kosten für Qualifikation und Berufsausbildung anfallen.

Die wachsende Nachfrage nach Computerprogrammen beeinflusst direkt die Nichtakzeptanz vieler Fachleute, dies kann auf nachteilige Weise für die Buchhaltung betrachtet werden, da es kein einziges System gibt, um die notwendigen Aktivitäten auszuführen, die die Buchhaltung benötigt.

Aber das Potenzial, das technologische Fortschritte für die Buchhaltungsberatung mit sich bringen, ist immens, und diese Potenziale können positive Auswirkungen auf die Akzeptanz des Einsatzes von Technologien zur Erreichung der Ziele im Rechnungswesen haben. In diesem Zusammenhang ist es so, dass Technologien die Gesamtansicht des Zählers erleichtern, in dem die Wahrnehmung einer geringeren Fehlerspanne und der Zugriff auf alle Daten von Interesse, die zusammengestellt und / oder abgerechnet werden können, in der Technologie einen Allpartner findet, um einen Exzellenten Dienst zu erbringen.

Es wird daher davon ausgegangen, dass die Studie ihr angegebenes Ziel erreicht und sich als Lücke zur Forschung bezieht, die auf der Grundlage der Rechnungslegungsberatung im Allgemeinen durchgeführt wird, was Möglichkeiten für die Idealisiertheit neuer Forschung eröffnet, indem dieses Thema auf eine eingeschränktere Form wie Finanz- oder sogar Management-Buchhaltungsberatung ausgeweitet wird.

\section{VERWEISE}

ALMEIDA, Leonardo Soares Francisco de et al. A Consultoria Contábil como Oportunidade de Negócios Profissionais-um Estudo Documental. Pensar Contábil, v. 20, n. $72,2018$.

RC: 93888

Disponível em: https://www.nucleodoconhecimento.com.br/business-administration- 
AL NATOUR, Jihad Rebhee Abdel Qader et al. The Impact of Information Technology on The Quality of Accounting Information (SFAC NO 8, 2010). Turkish Journal of Computer and Mathematics Education (TURCOMAT), v. 12, n. 13, p. 885-903, 2020.

AL-SHAFEAY, Karrar Mohammed; AL_DUJAILI, Mohammed Jawad; AL-WATTAR, Yasir Mohammed Ali. The impact of Information and Communication Technology (ICT) In the accounting system: advantages, applications, and challenges. PalArch's Journal of Archaeology of Egypt/Egyptology, v. 17, n. 6, p. 15814-15825, 2020.

ANGELIM, Vitória Ribeiro; BARRETO, Tayssa Vieira. As Contribuições da Contabilidade Consultiva: Um Estudo de Caso/The Contributions of Advisory Accounting: A Case Study. ID on line Revista de Psicologia, v. 14, n. 52, p. 317331, 2020.

ASUQUO, Akabom I.; DAN, Nicholas O.; EFFIONG, Glory T. Impact of information technology on accounting line of works. International Journal of Recent Technology and Engineering, v. 9, n. 2, p. 1572-1577, 2020.

DE ANDRADE SIMÕES, Maervelym Pâmella et al. Benefícios do uso da tecnologia Blockchain como instrumento para a auditoria contábil. Revista ambiente contábilUniversidade Federal do Rio Grande do Norte-ISSN 2176-9036, v. 13, n. 1, p. 3953, 2021.

ERCOLE, Flávia Falci; MELO, Laís Samara de; ALCOFORADO, Carla Lúcia Goulart Constant. Revisão integrativa versus revisão sistemática. Revista Mineira de Enfermagem, v. 18, n. 1, p. 9-12, 2014.

FERREIRA, Tiago Janes et al. Automação contábil: tecnologia em contabilidade sob a ótica da teoria institucional. XI Congresso ANPCONT. 3 a 6 de junho de 2017. Belo Horizonte, Minas Gerais.

RC: 93888

Disponível em: https://www.nucleodoconhecimento.com.br/business-administrationde/technologie-zu-fuehren 
GAFURI, Raquel Adriana Pin; ROJO, Cláudio Antonio; MIURA, Marcio Nakayama. Planejamento estratégico: diagnóstico e proposta de intervenção por meio de consultoria. Revista Capital Científico-Eletrônica (RCCe)-ISSN 2177-4153, v. 15, n. 1, p. 139-155, 2017.

MARION, JOSE CARLOS; RIBEIRO, OSNI MOURA. Introdução à contabilidade gerencial. Saraiva Educação SA, 2017. Disponível em: https://books.google.com.br/books?hl=pt-

$B R \& \mid r=\& i d=k D 1 n D w A A Q B A J \& o i=f n d \& p g=P T 5 \& d q=I n t r o d u \% C 3 \% A 7 \% C 3 \% A 30+\% C$ $3 \% \mathrm{~A} 0+$ contabilidade+gerencial.+\&ots=JjrSnq2mD0\&sig=vcUiQXgi1UkNhLanfgWU67 QVgas\#v=onepage\&q=Introdu\%C3\%A7\%C3\%A30\%20\%C3\%A0\%20contabilidade\% 20gerencial.\&f=false

SAMPAIO, Rosana Ferreira; MANCINI, Marisa Cotta. Estudos de revisão sistemática: um guia para síntese criteriosa da evidência científica. Brazilian Journal of Physical Therapy, v. 11, p. 83-89, 2007.

SCHIAVI, Giovana Sordi; BEHR, Ariel. Características dos diferentes modelos de negócios contábeis em relação às áreas da Contabilidade. REUNIR Revista de Administração Contabilidade e Sustentabilidade, v. 10, n. 3, p. 47-59, 2020.

SCHIAVI, Giovana Sordi et al. No caminho da inovação: análise das capacidades de inovação de empresas contábeis diante das tecnologias digitais. Revista Brasileira de Gestão de Negócios, v. 22, p. 381-405, 2020.

SILVA, Gustavo Oliveira et al. O impacto da tecnologia na profissão contábil sob perspectivas de pessoas com formação e/ou experiência profissional na área. Contribuciones a las Ciencias Sociales, n. 72, p. 3, 2020.

RC: 93888

Disponível em: https://www.nucleodoconhecimento.com.br/business-administration- 
SILVA, Fabiana Ferreira; RIBEIRO, Ana Regina Bezerra; SILVA, Washington Ferreira. Consultoría Organizacional como um Instrumento de Inovação em arranjos produtivos locais. Revista Organizações em Contexto, v. 14, n. 27, p. 29-52, 2018.

VILLA, Patricia et al. O monólogo contábil: uma análise do uso da contabilidade gerencial em micro e pequenas empresas à luz do processo comunicacional segundo Bakhtin". Monografia para obtenção de título de mestrado em contabilidade. UFPR, 2012.

XAVIER, Leonardo Montes; CARRARO, Wendy Beatriz Witt Haddad; RODRIGUES, Ana Tércia Lopes. Indústria 4.0 e avanços tecnológicos da área contábil: perfil, percepções e expectativas dos profissionais. Contexto. Porto Alegre, RS. Vol. 20, n. 45 (maio/ago. 2020), p. 34-50, 2020.

Eingereicht: Juli 2021.

Genehmigt: Juli 2021.

RC: 93888

Disponível em: https://www.nucleodoconhecimento.com.br/business-administration- 